\title{
Simulation of intrinsic resolution of scintillation camera in Monte Carlo environment
}

\begin{abstract}
In a typical scintillation camera system, intrinsic resolution is dependent upon the accuracy of the identification of an interaction position. This paper intends to set up a evaluation tool based on Monte Carlo simulation for the purpose of estimating the intrinsic resolution of scintillation cameras. Monte Carlo N-Particles (MCNP) Code was applied to simulate the components of the model platform referred to Toshiba GCA-7100A with a monolithic Sodium Iodide $(\mathrm{NaI})$ scintillator $(40 \times 40 \times 0.9525 \mathrm{~cm} 3)$. The simulation result was $3.7 \mathrm{~mm}$ full width at half maximum (FWHM) for intrinsic resolution which came out to be in a good agreement with the experimental result. This suggests that our proposed evaluation tool may help to optimize the parameters of the detector without physical experiments.
\end{abstract}

Keyword: Scintillation camera; MCNP; Nal; Intrinsic resolution 